\title{
A produção acadêmica acerca do uso de indicadores de desempenho para gestão hospitalar: uma abordagem bibliométrica
}

\section{Academic production about performance indicators usage in hospital management: A bibliometric approach.}

\author{
ALISSON DE MORAES BASTOS \\ SAMUEL VINÍCIUS BONATO \\ DÉBORA GOMES DE GOMES \\ Universidade Federal do Rio Grande
}

\section{RESUMO}

Este trabalho teve por objetivo apresentar uma caracterização da produção científica acerca de indicadores de desempenho em hospitais e organizações de cuidados à saúde, para tal, adotouse métodos de revisão oriundos da abordagem bibliométrica. A revisão baseou-se em um processo de seleção de artigos, a critério do pesquisador, que levou em consideração a análise dos títulos, resumos e conteúdos de 1109 artigos que compunham o conjunto encontrado. Para atingir o objetivo proposto escolheuse 31 artigos onde foram realizados quatro estudos, são eles: Produção, autoria e publicação, conteúdo, citações e referências utilizadas. Dentre os principais resultados encontrados destacase a nacionalidade dos autores, os autores mais utilizados para realização da fundamentação teórica na temática da pesquisa, os artigos de maior impacto acadêmico assim como revistas científicas de maior frequência e as metodologias mais utilizadas pelos pesquisadores.

Palavras-Chave

Indicadores de desempenho; Hospitais; revisão; 


\section{Abstract}

This paper aims to show a description of scientific production about performance indicators in hospitals and healthcare organizations, for this, review methodos originating in bibliometric approach were adopted. The review was based on a selection process of articles at the discretion of the researcher that took into account the analysis of the titles, abstracts and content of 1109 articles that composed the set found. In order to reach the proposed objective, 31 articles were selected, where four studies were carried out: Production, authorship and publication, content, citations and references used. Among the main results are listed the nationalities of the authors, the authors most used to carry out the theoretical foundation in the research theme, the articles with the greatest academic impact as well as scientific journals of higher frequencies and the metodologys most used by researchers.

Keywords: Performance indicators; Hospitals; Review.

\section{INTRODUÇão}

A Organização Mundial da Saúde (OMS) define os hospitais como elementos essênciais para o sistema de cobertura universal da saúde pois concentram recursos escassos em detrimento de uso efetivo dos mesmos, este é ocasionado pela prestação contínua de serviços complexos que são realizados através de encaminhamentos em rede planejados para atender a população (OMS, 2019). Para Silva, Borget e Schultz (2009) a complexidade dos serviços prestados é derivada da ampla oferta de serviços que podem se apresentar em forma de diagnósticos, tratamentos, hospedagem, ensino, pesquisa, entre outros. Os autores afirmam que para o hospital atuar com excelência se faz necessário o constante investimento em insalações físicas, materiais, conhecimento e equipamentos passíveis de gerenciamento.

No Brasil, a Constituição da República Federativa do Brasil garantiu o acesso á saúde como direito de todo cidadão e tornou a prestação de serviços em saúde uma obrigação do estado, assim como garante que o acesso a estes serviços se realize de maneira universal e igualitária (BRASIL, 1988). Apesar do aporte da legislação, colocar em prática o acesso a saúde de qualidade para população torna-se um processo de difícil gerenciamento graças a escassez de recursos oriun- 
dos do governo e pressões estabelecidas pelos planos de saúde. De acordo com Ramos et al. (2015), os hospitais brasileiros configuram-se como os principais provedores de serviço em saúde no país, com isto muitos gestores hospitalares optam, embora de forma precária, por sustentar as operações apenas na gestão dos custos, descartando ou pouco utilizando outras ferramentas de controle devido a impossibilidade de criação, manutenção e gerenciamento de sistemas mais caros e complexos de gestão (SILVA; BORGET; SCHULTZ, 2009).

Além dos processos de controle de custos, pode-se utilizar outros processos de gestão de resultados como a mensuração e análise do desempenho, que de acordo com Brignall e Balantine (1996), trata-se de uma forma de monitorar e interpretar os resultados das atividades organizacionais em função de garantir determinado objetivo. $\mathrm{O}$ investimento em sistemas de mensuração do desempenho pode trazer inúmero benefícios, pois de acordo com a OMS, a boa gestão das organizações hospitalares possibilita a oferta de atendimentos de alta qualidade, assim como o monitoramento das atividades interns e de agências reguladores que permitem o desenvolvimento de melhoria contínua dos processos (OMS, 2019).

Ao levar em consideração os aspectos já citados, o presente estudo, tem por objetivo descrever o atual estado da produção científica a respeito do tema de indicadores de desempenho em organizações hospitalares e de cuidados á saúde. Para Araújo e Alvarenga (2011) este tipo de pesquisa torna-se interessante pelo fato de haver uma incessante busca por mensurar, avaliar e caracterizar a ciência de modo a permitir a compreensão de um campo disciplinar. Neste sentido, para realização deste trabalho, foi delimitado uma série de objetivos específicos, são eles: (i) Descrever os dados com relação a autoria e produção dos artigos, (ii) demonstrar os temas de maior recorrência nas obras analisadas, (iii) levantar dados sobre a qualidade dos artigos utilizados, (iv) exibir as palavras-chave de maior recorrência, (v) demonstrar os artigos de maior impacto acadêmico através da análise das citações, (vi) levantar as instituições mais produtivas, (vii) realizar estudo com relação as citações e co-citações e (viii) analisar os trabalhos presentes nas referências bibliográficas dos artigos.

Este artigo está estruturado em cinco seções, após esta introdução é apresentada a fundamentação teórica a respeito das orga- 
nizações hospitalares, formas de gestão e o estudo do desempenho das mesmas, após está disposta a metodologia, onde se encontra a fundamentação do processo bibliométrico assim como a descrição do processo de coleta e análise de dados, na seção de resultados econtram-se os achados no processo de estudo de produção, autoria e publicação, análise de impacto, análise das citações, estudo das referências bibliográficas e estudo de conteúdo. A quinta seção traz as discussões e conclusões acerca das contribuições, implicações e limitações deste trabalho assim como sugestões para pesquisas futuras na temática da pesquisa.

\section{REFERENCIAL TEÓRICO}

\subsection{Serviços em saúde e organizações hospitalares}

A prestação de serviço na área da saúde configura-se como um tema explorado há algumas décadas, esta apresenta características complexas e contingenciais onde se faz necessário levar em conta aspectos que tem relação com a maturidade da organização, ao tipo de serviço prestado e os clientes ou pacientes que estão em atendimento. As organizações prestadoras destes serviços precisam estar atentas ao tipo atendimento realizado, pois este apresenta características variáveis de acordo com dimensões geográficas, demográficas, biofísicas e psicossociais dos pacientes, além de adaptar-se aos padrões exigidos por agências regulamentadoras e da autonomia inerente ao profissional da área (DUSSAULT, 1992). No Brasil, de acordo com Ramos et al. (2015) os hospitais são os maiores concentradores de serviços hospitalares apresentando um quarto das consultas e 80\% das internações além de participação em $60 \%$ dos gastos do setor. Os hospitais podem ser classificados por diversos vieses como seu modo de estruturação, suas operações, porte, entre outros.

Os hospitais são classificados por Mintzberg (2006), em sua análise estrutural, como burocracias profissionais, onde os trabalhadores envolvidos na prestação de serviço, em muitas tarefas, desfrutam de descentralização e autonomia em suas ações enquanto outros experimentam de tarefas padronizadas, estáveis e previsíveis. Neste tipo de estrutura, o profissional trabalha livre e de frente ao consumidor do serviço de modo a proporcionar maior variabilidade na prestação. 
Com relação ao porte, a maioria dos hospitais brasileiros configuram-se como de pequeno porte, estes descentralizados e regionalizados experimentam de baixos índices de eficiência. Já hospitais de grande porte conseguem apresentar melhores resultados devido maior poder de barganha, maiores índices de ocupação, amortização de custos fixos, entre outros. O porte do hospital pode ser definido pelo número de operações ou pelo número de leitos disponíveis (RAMOS et al., 2015).

As operações deste tipo de organização caracterizam-se pela flexibilidade, de acordo com Alolayyan, Ali e Idris (2012) a flexibilidade operacional se faz necessário devido duas necessidades de reação, a primeira diz respeito aos fatores externos que afetam a organização e a segunda gira em torno das contingencias de serviço apresentadas por cada paciente.

É importante destacar que os hospitais apresentam algumas demandas peculiares no que diz respeito á suas finanças, os mesmos têm de lidar com a escassez de recursos oriundos do governo, com o alto custo das operações e com pressões estabelecidas pelos planos de saúde para o custeio dos serviços prestados e da continuidade dos convênios (SOUZA et al., 2010).

Todas as características citadas até este momento se relacionam com as formas como as organizações hospitalares buscam sua principal fonte de resultado que é a satisfação do paciente, para Lovaglio e Vittadini (2015) esta é afetada por diversos agentes oriundos da qualidade percebida, para os autores, esta tem por finalidade a conquista da confiabilidade do paciente que para Aksezer (2011) esta representa o mais alto grau de satisfação. As características apresentadas podem ser mensuradas e gerenciadas de modo que se faz necessário o uso de ferramentas gerenciais para controlar as variáveis que afetam estas características, no próximo subtítulo será abordado a questão do desempenho, dos sistemas de mensuração e o uso de indicadores para avaliar organizações hospitalares.

\subsection{DESEMPENHO, MENSURAÇÃo E INDICADORES}

As organizações demandam de ferramentas que expressem a efetividade de suas operações, neste modo faz-se necessário gerenciar as atividades internas de modo á controla-las para gerar resulta- 
do, estes resultados refletirão o desempenho organizacional. Embora o desempenho apresente um domínio mais restrito da organização, este possui características multidimensionais que determinarão seu delineamento, pode-se destacar entre outras o entendimento de quem são as partes interessadas no negócio, o panorama de desempenho adotado e o tipo de organização (RICHARD et al., 2009).

Medir o desempenho configura-se como uma característica essencial dentro das organizações (SANTOS; QUEL; VIEIRA; ROSINI, 2019), de modo que permite a posse de dados consolidados acerca de seu andamento operacional, de acordo com Petri (2005), estes dados são extremamente exigidos pelo mercado, pois possibilitam a identificação de falhas e o redesenho das estratégias implementadas. O desempenho é expresso por indicadores, Kaplan e Norton (1997) definem os indicadores de desempenho como instrumentos gerenciais que compõem sistemas de mensuração de modo que expressão de maneira quantitativa medidas de eficiência e eficácia do que está sendo medido. Os mesmos relatam as metas organizacionais e proporcionam uma reflexão das ações gerenciais.

Quando se trata de hospitais, se faz necessário desenvolver indicadores que relatem as peculiaridades destas organizações, de modo de que devem refletir resultados relacionados com as necessidades dos médicos, pacientes, enfermeiros e demais atores envolvidos na prestação de serviço. No Brasil, os registros de sistemas de mensuração de desempenho formados por indicadores surgiram na década de 90, o primeiro coordenado pelo Comitê de Qualidade Hospitalar da Associação Paulista de Medicina $(\mathrm{CQH})$, seguido pelo Sistema Padronizado para Gestão Hospitalar (SIPAGEH) da Unisinos no Rio Grande do Sul, entretanto estes sistemas de mensuração levam em consideração indicadores utilizados pelo Sistema Único de Saúde (SUS) para avaliação da efetividade dos recursos repassados pelo governo federal o que torna a utilidade destes indicadores limitada (SCHOUT; NOVAES, 2007).

Além dos sistemas específicos de mensuração do desempenho, outro conjunto de indicadores utilizados com frequência na área da saúde são os indicadores financeiros, que são essenciais para todo tipo de organização, de acordo com Souza et al. (2010), estes indicadores são de fácil levantamento devido serem amplamente divulga- 
dos pelos hospitais e levarem em consideração valores monetários para mensuração e são excelentes instrumentos de comprovação da efetividade do emprego de recursos, principalmente dos oriundos do SUS, além de proporcionarem a comparação entre organizações e períodos de tempo. Cabe ressaltar que para organizações hospitalares que não possuem fins lucrativos os indicadores de lucro e rentabilidade podem não ser tão expressivos.

Embora estes sistemas relacionados ao SUS e as finanças atuem de forma limitada, Ramos et al. (2015) afirmam que existem pelo mundo outros sistemas que visam avaliar indicadores mais sensíveis à atividade hospitalar, como a efetividade clínica, foco no paciente e nos profissionais, mas que estes se tornam de difícil mensuração pela escassez de dados, de acordo com os autores os sistemas de mensuração acabam priorizando a avaliação da movimentação dos pacientes e a ocupação dos leitos.

\section{Procedimentos metodológicos}

Este trabalho é classificado como uma pesquisa bibliográfica, nas definições de Gil (2010), este tipo de pesquisa é elaborada com material já publicado e utiliza como objetivo a "identificação do estágio atual do conhecimento referente ao tema" (GIL, 2010, p. 30), neste tipo de pesquisa o portfólio de análise é composto por material impresso e digital presente em revistas, livros, anais de eventos, teses, dissertações, jornais etc. Segundo argumenta Vieira (2007), trata-se de mapeamento da produção a respeito de determinado tema.

Neste estudo buscou-se estudar e caracterizar a produção científica acerca dos indicadores de desempenho e, para tal, foi realizada busca por artigos publicados em bases de dados, para isto, utilizou-se o método da bibliometria que, de acordo com Caldas e Tinoco (2004) é oriundo da junção de métodos da Biblioteconomia e da Ciência da Informação e busca analisar a estrutura de um determinado conhecimento, assim como o comportamento de publicação dos pesquisadores de determinada área.

Para coletar os artigos relacionados ao tema de pesquisa delimitou-se três bases de dados, são elas: Google Scholar, Scopus e Science Direct, as buscas nas mesmas foram realizadas por dois termos de pesquisa relacionadas á artigos, títulos, resumos e palavras-chave e os resultados podem ser conferidos na tabela 01 . 
Tabela 01: Termos e buscas nas Bases de dados.

\begin{tabular}{l|l|l|l|l}
\hline Pesquisa & Termos procurados & $\begin{array}{l}\text { Google } \\
\text { Scholar }\end{array}$ & Scopus & $\begin{array}{l}\text { Science } \\
\text { Direct }\end{array}$ \\
\hline 1 & $\begin{array}{l}\text { PERFORMANCE INDICATOR } \\
\text { and (HOSPITALS or HEAL- } \\
\text { THCARE). }\end{array}$ & 16.400 & 362 & 97 \\
\hline 2 & $\begin{array}{l}\text { (PERFORMANCE MEASUR- } \\
\text { MENT or EVALUATION) and } \\
(\text { (HEALTHCARE or HOSPI- } \\
\text { TAL) not HOSPITALITY). }\end{array}$ & 23.000 & 602 & 48 \\
\hline
\end{tabular}

Fonte: Autores da pesquisa

É possível observar na tabela 01 que o Google Scholar foi a base de dados que apresentou maior número de resultados, mas cabe ressaltar que nesta não foram aplicados filtros de pesquisa. Nas bases de dados Scopus e Science foram utilizados alguns filtros á critério dos pesquisadores, são eles: Artigos compreendidos entre 2007 e 2017, limitados a língua inglesa e nas áreas de conhecimento Ciências Sociais e Negócios, Gestão e Contabilidade.

O processo de composição do portifólio consistiu em três etapas. A primeira parte do processo consistiu em mesclar as buscas a fim de verificar a relevância dos artigos encontrados, a etapa seguinte se constituiu em refinar os achados para definir os artigos mais qualificados para a composição do portifólio. Ao mesclar as coletas de dados, se fez necessário avaliar a quantidade de artigos em duplicidade para excluir os pares da composição, realizou-se a leitura do título, do resumo e do texto completo. Por fim, realizou-se consulta a plataforma Qualis da Coordenação de Aperfeiçoamento de Pessoal de Nível Superior (Capes) com intenção de excluir do grupo de artigos aqueles que foram publicados em periódicos que não possuem fator de impacto para a área Administração, Ciências contábeis e Turismo.

A análise dos dados se deu através do viés quantitativo, pois seus achados representam dados numéricos passíveis de análise estatística, entratanto ao realizar a análise dos temas e metodos empregados optou-se por realizar uma análise de conteúdo, que é uma abordagem qualitativa orientada por Bardin (2011). Todas as ferramentas bibliométrica adotadas estão presentes no quadro 01. 
Quadro 01: Ferramentas bibliométricas e métodos de análise.

\begin{tabular}{|l|l|l|}
\hline Ferramenta & Análise & Autor \\
\hline $\begin{array}{l}\text { Produção, } \\
\text { Autoria e } \\
\text { Publicação }\end{array}$ & $\begin{array}{l}\text { Análise do estrato qualis, dos autores, } \\
\text { dos periódicos, do ano de publicação } \\
\text { e das instituições envolvidas através } \\
\text { de contagem e análise de frequência } \\
\text { simples (fi). }\end{array}$ & Wormell (1998) \\
\hline Citações & $\begin{array}{l}\text { Análise do impacto acadêmico dos } \\
\text { artigos observado através do núme- } \\
\text { ro de citações presentes em bases de } \\
\text { dados. A análise se deu pela soma } \\
\text { das citações e análise de frequência } \\
\text { simples (fi). }\end{array}$ & Wanti (2002) \\
\hline $\begin{array}{l}\text { Referências } \\
\text { Bibliográficas }\end{array}$ & $\begin{array}{l}\text { Análise dos trabalhos citados ao longo } \\
\text { do texto. Engloba-se autores, ano de } \\
\text { publicação, periódicos e tipo de tra- } \\
\text { balho. Foram utilizadas ferramentas } \\
\text { de contagem e análise de frequeência } \\
\text { simples (fi). }\end{array}$ & Wormell (1998) \\
\hline Conteúdo & $\begin{array}{l}\text { Análise qualitativa acerca do conteú- } \\
\text { do dos artigos, feito através de cate- } \\
\text { gorias de análise que emergiram da } \\
\text { leitura dos mesmos. Os artigos inse- } \\
\text { ridos nas categorias foram quantifica- } \\
\text { dos e a análise se deu por frequência } \\
\text { simples (fi). }\end{array}$ & \\
\hline
\end{tabular}

Fonte: Autores.

As ferramentas presentes no quadro 01 tem uso recorrente no âmbito das Ciências Sociais Aplicadas nos últimos anos como é possível observar em Silva e Miranda (2018) que adotaram estas ferramentas para descrever artigos acerca da gestão do conhecimento no setor público, em Longaray et al. (2018) que as utilizaram para caracterizar a literatura acerca de propostas de redesenho de processos e o papel das equipes organizacionais e em Pereira Jr., D'avila e Pereira (2018) que adotaram as mesmas ferramentas para mapear a produção científica acerca de indicadores de desempenho em serviços e produção. 


\section{RESULTADOS E DISCUSSÕES}

O estudo dos dados levantados foi realizado em quatro etapas distintas, na primeira etapa avaliou-se os dados de produção, autoria e publicação, em seguida explorou-se o conteúdo dos artigos, em um terceiro momento foi feito um estudo a respeito das citações dos artigos listados e, por fim, realizou-se o estudo das referências bibliográficas.

\subsection{Estudo de produção, autoria e publicação}

Nesta etapa, em primeiro lugar observou-se o fator de impacto dos periódicos em que os artigos foram publicados, de acordo com a tabela Qualis da Capes para a área Administração, Ciências Contábeis e Turismo. Destaca-se que 68\% dos artigos foram publicados em periódicos do estrato A1, seguido de $23 \%$ pertencente ao estrato A2 e outros $9 \%$ publicados em periódicos dos outros estratos, isto revela que os artigos que pertenceram ao portifólio foram publicados em revistas de alto nível, de acordo com a Capes.

Apareceram nos dados 19 periódicos, destes destaca-se o Service Industries Journal, o International Journal of Business Innovation and Research e o International Journal of Productivity and Performance Management. Os periódicos que apresentaram mais de 1 artigo no portifólio estão dispostos na tabela 02 .

Tabela 02: Periódicos em que os artigos foram publicados

\begin{tabular}{l|l|l|l}
\hline Periódicos & Estrato & Frequência & $f \mathbf{~}$ \\
\hline Service Industries Journal & $\mathrm{A} 1$ & 5 & 0,1613 \\
\hline $\begin{array}{l}\text { International Journal of Business Innovation } \\
\text { and Research }\end{array}$ & $\mathrm{A} 2$ & 3 & 0,0968 \\
\hline $\begin{array}{l}\text { International Journal of Productivity and Per- } \\
\text { formance Management }\end{array}$ & $\mathrm{A} 1$ & 3 & 0,0968 \\
\hline Expert Systems with Applications & $\mathrm{A} 1$ & 2 & 0,0645 \\
\hline $\begin{array}{l}\text { International Journal of Business Performance } \\
\text { Management }\end{array}$ & $\mathrm{A} 2$ & 2 & 0,0645 \\
\hline Journal of Modelling in Management & $\mathrm{B} 2$ & 2 & 0,0645 \\
\hline Evaluation and Program Planning & $\mathrm{A} 1$ & 2 & 0,0645 \\
\hline Total de periódicos & 19 & & \\
\hline
\end{tabular}

Fonte: Dados da pesquisa. 
É possível verificar, na tabela 2, que os três primeiros periódicos representam mais de um terço dos artigos selecionados para o portifólio.

Quanto a distribuição continental pode-se afirmar que $42 \%$ dos autores são europeus e 38\% são asiáticos. O resumo da distribuição de autores de acordo com os continentes de origem está resumido na tabela 03.

Tabela 03: Distribuição de autores por continente

\begin{tabular}{l|l|l}
\hline Continentes & Autores & fi \\
\hline Europa & 42 & 0,4242 \\
\hline Ásia & 38 & 0,3838 \\
\hline Oceania & 8 & 0,0808 \\
\hline América do Norte & 7 & 0,0707 \\
\hline América do Sul & 4 & 0,0404 \\
\hline Total & 99 & 0,9999 \\
\hline
\end{tabular}

Fonte: Dados da pesquisa

A tabela 3 demonstra que dentro do portifólio apresentam-se 99 autores distintos distribuídos por 4 continentes. Após analisar a distribuição continental é possível verificar a nacionalidade dos os autores dos artigos, neste sentido cabe destacar os 10 países de maior recorrência (tabela 04).

Tabela 04: Nacionalidade dos autores estudados

\begin{tabular}{l|l|l}
\hline Nacionalidades & Autores & fi \\
\hline Itália & 16 & 0,1616 \\
\hline Alemanha & 9 & 0,0909 \\
\hline Malásia & 8 & 0,0808 \\
\hline Taiwan & 8 & 0,0808 \\
\hline Índia & 6 & 0,0606 \\
\hline Turquia & 7 & 0,0707 \\
\hline Canadá & 5 & 0,0505 \\
\hline Reino Unido & 5 & 0,0505 \\
\hline Brasil & 4 & 0,0404 \\
\hline Fontemburgo & 4 & 0,0404
\end{tabular}

Fonte: Dados da pesquisa 
É possível observar na tabela 04 que os países de maior frequência foram Itálisa, Alemanha e Malásia que juntos representam cerca de $33 \%$ dos autores encontrados. Após verificar a nacionalidadede dos autores, foi verificado também quais instituições estes representavam, localizou-se 49 instituições e as com maior frequência estão destacadas na tabela 05 .

Tabela 05: Instituições com mais autores

\begin{tabular}{l|l|l}
\hline Instituições & Autores & fi \\
\hline University Of Cologne & 7 & 0,0707 \\
\hline University Pendidikan Sultan Idris & 5 & 0,0505 \\
\hline Federal University Of Minas Gerais & 4 & 0,0404 \\
\hline
\end{tabular}

Fonte: Dados da pesquisa

Na continuidade da análise do conteúdo dos artigos, foi feito estudo acerca das palavras-chave utilizadas pelos autores dos artigos, localizou-se um total de 94 palavras-chave, configurando média de 3 por artigo. A figura 1 demonstra a nuvem de palavras formada pelas palavras-chave.

Figura 01: Word Cloud de palavras-chave

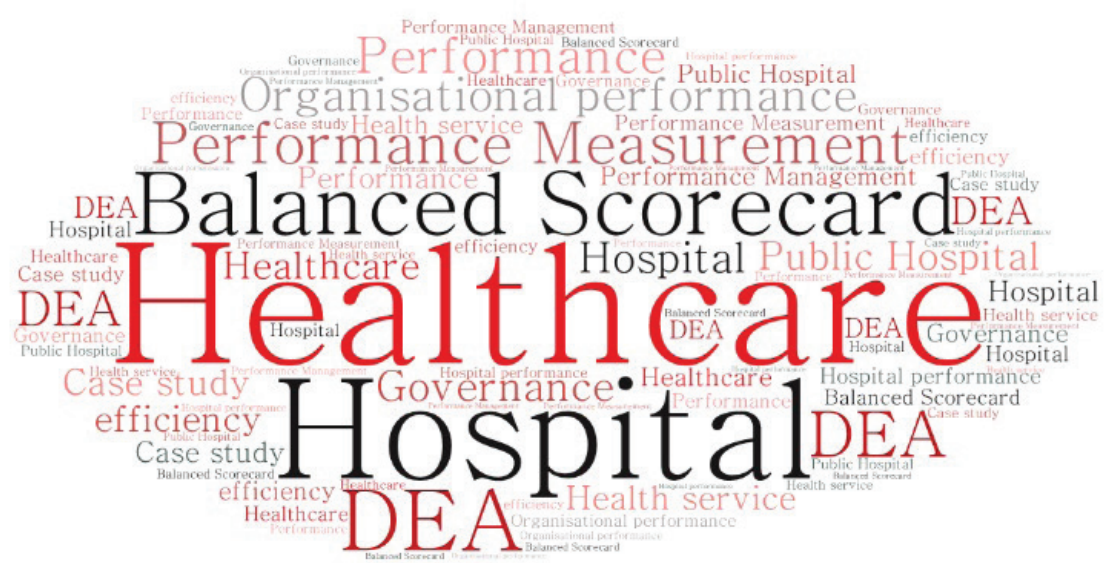

Fonte: Autores da pesquisa 
As palavras-chave de maior recorrência foram os termos healthcare e Balance Scorecard ambos apareceram em 7 artigos configurando $22,58 \%$ sobre o total de artigos, na segunda posição aparecem os termos hospitals e performance measurement frequente em 6 artigos $(19,35 \%)$.

\section{2 Estudo das citações}

A terceira parte da análise bibliométrica dedicou-se a levantar dados sobre o impacto destes artigos na comunidade acadêmica, nesse sentido, levantou-se as citações dos artigos selecionados, nesta etapa foi realizado consulta nas plataformas Google Scholar e Web of Science em 25 de maio de 2018 para verificar a quantidade de vezes que cada artigo foi citado em outros documentos. A tabela 06 traz os artigos que mais foram citados somando-se as duas plataformas.

Tabela 06: Trabalhos de maior repercussão

\begin{tabular}{l|l|l|l|l}
\hline Artigo & Título & $\begin{array}{l}\text { Goo- } \\
\text { gle } \\
\text { Scho- } \\
\text { lar }\end{array}$ & $\begin{array}{l}\text { Web } \\
\text { of } \\
\text { Scien- } \\
\text { ce }\end{array}$ & Total \\
\hline 22 & $\begin{array}{l}\text { GRIGOROUDIS, E.; ORFANOUDAKI, E.; } \\
\text { ZOPOUNIDIS, C. Strategic performance } \\
\text { measurement in a healthcare organisa- } \\
\text { tion: A multiple criteria approach based } \\
\text { on balanced scorecard. Omega, 2012. }\end{array}$ & 236 & 67 & 303 \\
\hline 17 & $\begin{array}{l}\text { KELMAN, S.; FRIEDMAN, J. N. Perfor- } \\
\text { mance improvement and performance } \\
\text { dysfunction: An empirical examination of } \\
\text { distortionary impacts of the emergency } \\
\text { room wait-time target in the English Na- } \\
\text { tional Health Service. Journal of Public } \\
\text { Administration Research and Theory, } \\
\text { 2009. }\end{array}$ & $\begin{array}{l}\text { GURD, B.; GAO, T. Lives in the balance: } \\
\text { an analysis of the balanced scorecard } \\
\text { (BSC) in healthcare organizations. In- } \\
\text { ternational Journal of Productivity and } \\
\text { Performance Management, 2007. }\end{array}$ & 107 & 0 \\
\hline
\end{tabular}




\begin{tabular}{|c|c|c|c|c|}
\hline 11 & $\begin{array}{l}\text { TSAI, H. Y.; CHANG, C. W.; LIN, H. L. } \\
\text { Fuzzy hierarchy sensitive with Delphi } \\
\text { method to evaluate hospital organiza- } \\
\text { tion performance. Expert Systems with } \\
\text { Applications, 2010. }\end{array}$ & 60 & 31 & 91 \\
\hline 15 & $\begin{array}{l}\text { LIN, Q. L.; LIU, L.; LIU, H. C. \& WANG, } \\
\text { D. J. Integrating hierarchical balan- } \\
\text { ced scorecard with fuzzy linguistic for } \\
\text { evaluating operating room performance } \\
\text { in hospitals. Expert Systems with Appli- } \\
\text { cations, } 2013 \text {. }\end{array}$ & 46 & 17 & 63 \\
\hline 7 & $\begin{array}{l}\text { NUTI, S.; SEGHIERI, C.; VAINIERI, M. } \\
\text { Assessing the effectiveness of a performan- } \\
\text { ce evaluation system in the public health } \\
\text { care sector: Some novel evidence from the } \\
\text { Tuscany region experience. Journal of Ma- } \\
\text { nagement and Governance, } 2013 \text {. }\end{array}$ & 52 & 0 & 52 \\
\hline 14 & $\begin{array}{l}\text { ZIGAN, K.; MACFARLANE, F.; DESOM- } \\
\text { BRE, T. Intangible resources as perfor- } \\
\text { mance drivers in European hospitals. } \\
\text { International Journal of Productivity } \\
\text { and Performance Management, } 2007 . \\
\end{array}$ & 50 & 0 & 50 \\
\hline 12 & $\begin{array}{l}\text { AUBRY, M.; RICHER, M. C.; LAVOIE- } \\
\text { TREMBLAY, M. Governance performance } \\
\text { in complex environment: The case of } \\
\text { a major transformation in a university } \\
\text { hospital. International Journal of Project } \\
\text { Management, 2014. }\end{array}$ & 36 & 10 & 46 \\
\hline 5 & $\begin{array}{l}\text { WU, C.R.; CHANG, C. W. \& LIN, H. L. } \\
\text { An organisational performance measu- } \\
\text { rement model based on AHP sensitivity } \\
\text { analysis Hung-Lung Lin. Int. J. Business } \\
\text { Performance Management, v. 9, n. 1, p. } \\
\text { 77-91, } 2007 .\end{array}$ & 29 & 0 & 29 \\
\hline 2 & $\begin{array}{l}\text { EIRIZ, V.; BARBOSA, N.; FIGUEIREDO, } \\
\text { J. A conceptual framework to analyse } \\
\text { hospital competitiveness. Service Indus- } \\
\text { tries Journal, } 2010 .\end{array}$ & 22 & 6 & 28 \\
\hline
\end{tabular}

Fonte: Dados da Pesquisa 
Ao visualizar-se a Tabela 6, nota-se que o artigo 22 foi o de maior repercussão, com 303 citações, destaca-se que esta referência se constitui em um indicativo para a literatura da temática analisada.

\subsection{Estudo das referências bibliográficas}

Nesta etapa da análise bibliométrica, foi realizado estudo dos trabalhos e autores presentes nas referências bibliográficas. A soma de citações de todos os artigos chegou a 3.165 e configurou-se uma média de 102 citações. Com relação as obras citadas, foi identificado o total de 1.425 , as 10 obras de maior citação podem ser conferidas na tabela 07.

Tabela 07: Obras de maior frequência nas referências bibliográficas

\begin{tabular}{l|l|l}
\hline Título da obra & Autor & $\begin{array}{l}\text { Cita- } \\
\text { ções }\end{array}$ \\
\hline The Analytic Hierarchy Process. & Saaty, T. L. & 3 \\
\hline $\begin{array}{l}\text { The Balanced Scorecard: Translating Strategy } \\
\text { into Action. }\end{array}$ & Kaplan, R. S. & 3 \\
\hline $\begin{array}{l}\text { Performance measurement system design: a } \\
\text { literature review and research agenda. }\end{array}$ & Neely, A. & 3 \\
\hline Case Study Research: Design and Methods. & Yin, R. K. & 3 \\
\hline $\begin{array}{l}\text { The association between hospital ownership } \\
\text { and technical efficiency in a managed care } \\
\text { environment. }\end{array}$ & Lee, K. H. & 2 \\
\hline $\begin{array}{l}\text { The Concept of Corporate Strategy. } \\
\begin{array}{l}\text { The balanced scorecard: a potent tool for } \\
\text { energizing and focusing healthcare organiza- } \\
\text { tion management. }\end{array}\end{array}$ & Chow, C. W. & 2 \\
\hline $\begin{array}{l}\text { The balanced scorecard and intangible assets: } \\
\text { similar ideas, unaligned concepts. }\end{array}$ & Marr, B. & 2 \\
\hline Why measurement initiatives fail. & Neely, A. & 2 \\
\hline Use of the balanced scorecard in healthcare. & Zelman, W.N. & 2 \\
\hline
\end{tabular}

Fonte: Dados da pesquisa 
É possível verificar na tabela 07 que nenhuma obra ultrapassou a marca de 3 citações. Das obras citadas, foram identificados 10 tipos de obras, dentre eles pode-se citar livros, periódicos, teses e dissertações, estes dados podem ser conferidos na tabela 8.

Tabela 08: Tipos de obras identificadas

\begin{tabular}{l|l|l}
\hline Tipo de trabalho & Frequência & Fi \\
\hline Artigo de periódico & 1071 & 0,7516 \\
\hline Livro & 220 & 0,1544 \\
\hline Relatório Institucional & 76 & 0,0533 \\
\hline Paper de conferência & 36 & 0,0253 \\
\hline Jornal de circulação & 6 & 0,0042 \\
\hline Dissertação & 4 & 0,0028 \\
\hline Tese & 4 & 0,0028 \\
\hline Artigo de site & 4 & 0,0028 \\
\hline Manual & 3 & 0,0021 \\
\hline Trabalho de conclusão de curso & 1 & 0,0007 \\
\hline Fonte: Dados da pesquisa & &
\end{tabular}

A tabela 08 demonstra que as obras de maior recorrência, cerca de três quartos, são artigos publicados em periódicos, o que demonstra que este recurso é o mais utilizado pelos pesquisadores do tema da pesquisa, seguido pelos livros. Este resultado demonstra uma inversão dos achados de Santos, Lima e Martins (2009) onde foi apontado que as principais fontes de fundamentação teórica eram os livros nacionais e seguido das revistas internacionais.

Em detrimento dos artigos de periódicos terem aparecido em destaque na fundamentação teórica das pesquisas optou-se por dispor periódicos de maior frequência nas referências bibliográficas (tabela 09). 
Tabela 09: Periódicos de maior frequência nas referências bibliográficas

\begin{tabular}{l|l|l}
\hline Periódico & Frequência & fi \\
\hline European Journal of Operational Research & 18 & 0,0271 \\
\hline Harvard Business Review & 14 & 0,0211 \\
\hline Journal of Health Care Finance & 13 & 0,0196 \\
\hline Health Policy & 12 & 0,0181 \\
\hline Omega & 11 & 0,0166 \\
\hline The Service Industries Journal & 11 & 0,0166 \\
\hline Health Services Research & 10 & 0,0151 \\
\hline Strategic Management Journal & 9 & 0,0136 \\
\hline Journal of Operations Management & 9 & 0,0136 \\
\hline Management Science & 9 & 0,0136 \\
\hline
\end{tabular}

Fonte: Dados da pesquisa

É possível observar, com auxílio da tabela 09, que os periódicos de maior referência pelos pesquisadores da temática da pesquisa são o European jornal of operational research, o Harvard business review e o Journal os health care finance, todos internacionais, estes agregam $6,7 \%$ das obras publicadas em periódicos referenciadas e levando em consideração um total de 663 periódicos identificados.

Com relação a autoria das obras citadas, foram identificados 2.594 autores e os 15 autores com maior número de citações estão presentes na tabela 10 .

Pode-se verificar na tabela 10, que os autores Kaplan, R. S. e Norton, D. P. foram os autores mais utilizados pelos pesquisadores como referência em seus trabalhos, com mais de 20 citações cada.

Por fim, realizou-se a análise do ano de publicação das obras referenciadas, o gráfico 1 mostra a quantidade de obras e faz uma relação com a década em que foram publicadas. 
Tabela 10: Autores com maior número de citações nas referências bibliográficas

\begin{tabular}{l|l|l}
\hline Autor & Citações & $f \mathbf{i}$ \\
\hline Norton, D.P. & 32 & 0,0101 \\
\hline Kaplan, R.S. & 29 & 0,0092 \\
\hline Neely, A. & 15 & 0,0047 \\
\hline Ozcan, Y.A. & 10 & 0,0032 \\
\hline Charnes, A. & 9 & 0,0028 \\
\hline Siskos, Y. & 7 & 0,0022 \\
\hline Van de Ven, A.H. & 6 & 0,0019 \\
\hline Mintzberg, H. & 6 & 0,0019 \\
\hline Kennerley, M. & 6 & 0,0019 \\
\hline Donabedian, A. & 6 & 0,0019 \\
\hline Cooper, W.W. & 6 & 0,0019 \\
\hline Aubry, M. & 6 & 0,0019 \\
\hline Bourne, M. & 6 & 0,0019 \\
\hline Saaty, T.L. & 5 & 0,0016 \\
\hline Venkatraman, N. & 5 & 0,0016 \\
\hline Fon Dados pesqi6
\end{tabular}

Fonte: Dados da pesquisa

Gráfico 01: Década de publicação das obras referenciadas

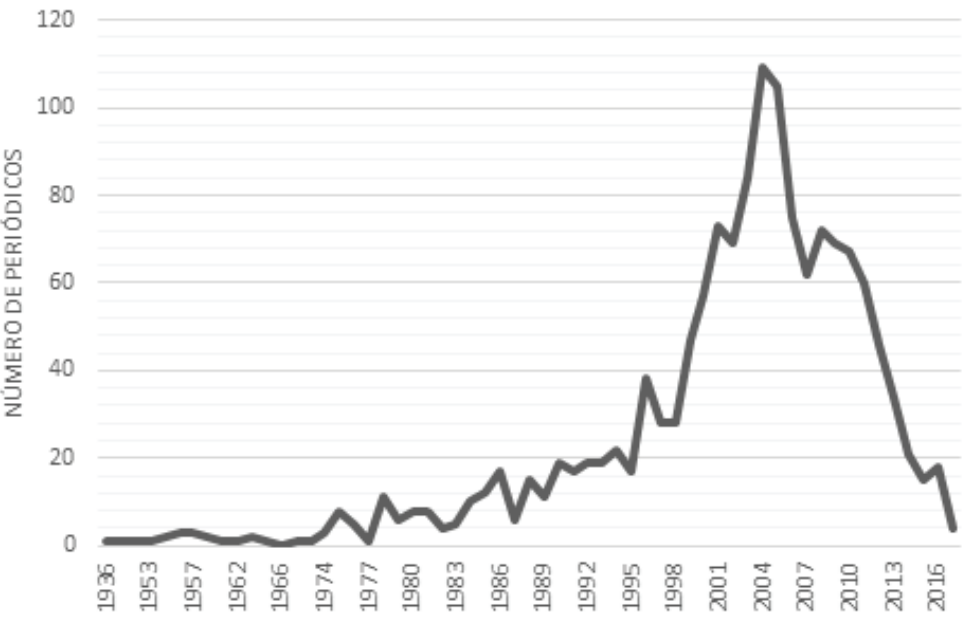

Fonte: Dados da pesquisa 
É possível verificar que as datas de publicação das obras referenciadas concentram-se entre 1995 e 2010, sendo os anos 2004 e 2005 os anos mais recorrentes, algumas obras, as mais clássicas, localizam-se entre a década de 30 e os anos 90 e por fim acentua-se uma diminuição no número de obras quando se leva em consideração os últimos 10 anos.

\section{4 Estudo de conteúdo}

Na segunda etapa de análise bibliométrica, realizou-se a análise de conteúdo dos artigos, em primeiro lugar foi realizado o estudo dos temas apresentados nos artigos, foram identificados 28 categorias de temas distintos e os temas que apresentaram frequência maior ou igual a 2 estão expressos na tabela 11.

Tabela 11: Temas de maior recorrência

\begin{tabular}{l|l|l|l}
\hline Categorias & Conteúdo & $\begin{array}{l}\text { No } \\
\text { arti- } \\
\text { gos }\end{array}$ & fi \\
\hline $\begin{array}{l}\text { Análises de } \\
\text { desempenho }\end{array}$ & $\begin{array}{l}\text { Estudos acerca da análise do } \\
\text { desempenho de hospitais e ins- } \\
\text { tituições na área da saúde com } \\
\text { análises críticas envolvendo o uso } \\
\text { da literatura. }\end{array}$ & 11 & 0,3548 \\
\hline $\begin{array}{l}\text { Mensuração de } \\
\text { desempenho }\end{array}$ & $\begin{array}{l}\text { Estudos acerca de descrições } \\
\text { numéricas acerca do desempenho } \\
\text { de hospitais e instituições da área } \\
\text { da saúde. }\end{array}$ & 6 & 0,1935 \\
\hline $\begin{array}{l}\text { Uso do Balance } \\
\text { Scorecard (BSC) }\end{array}$ & $\begin{array}{l}\text { Estudos que debatem o uso da } \\
\text { ferramenta de BSC na área da } \\
\text { saúde. }\end{array}$ & 5 & 0,1612 \\
\hline $\begin{array}{l}\text { Eficiência dos } \\
\text { recursos; }\end{array}$ & $\begin{array}{l}\text { Estudos que investigam o uso dos } \\
\text { recursos hospitalares. }\end{array}$ & 2 & 0,0645 \\
\hline $\begin{array}{l}\text { Inovação e de- } \\
\text { sempenho }\end{array}$ & $\begin{array}{l}\text { Estudos que envolvem questões } \\
\text { que se relacionam com a inovação } \\
\text { que englobam o desempenho. }\end{array}$ & 2 & 0,0645 \\
\hline
\end{tabular}

Fonte: Dados da pesquisa 
É possível observar na tabela 11 que os temas mais recorrentes no período compreendido entre 2007 e 2017, foram a avaliação de desempenho na área da saúde, presente em 35,48\% dos artigos, estes artigos visavam avaliar e descrever análises acerca do desempenho de organizações da área da saúde, em seguida a medição de desempenho em organizações hospitalares, presente no conteúdo de 19,35\% dos artigos que preocupavam-se em levantar expressões numéricas acerca do desempenho e o uso do Balance Scorecard, presente em $16,12 \%$ dos artigos, tais artigos expressavam formas e importância do uso da ferramenta em organizações hospitalares.

Em um segundo momento, foram analisadas as principais características metodológicas dos artigos do portifólio e foram reconhecidas 19 categorias distintas e as que possuem frequência maior ou igual a 2 estão presentes na tabela 12 .

\section{Tabela 12: Médotos de maior recorrência}

\begin{tabular}{l|l|l|l}
\hline Categorias & Conteúdo & $\begin{array}{l}\mathbf{N} \text { o } \\
\text { arti- } \\
\text { gos }\end{array}$ & fi \\
\hline $\begin{array}{l}\text { Questionário } \\
\text { estruturado }\end{array}$ & $\begin{array}{l}\text { Estudos que para chegar em seu } \\
\text { resultado utilizaram de questionários } \\
\text { para coleta de dados }\end{array}$ & 09 & 0,2903 \\
\hline $\begin{array}{l}\text { Análise Envol- } \\
\text { tória de dados } \\
\text { (DEA) }\end{array}$ & $\begin{array}{l}\text { Estudos que realizaram do método } \\
\text { de DEA para a análise de seus resul- } \\
\text { tados. }\end{array}$ & 5 & 0,1612 \\
\hline $\begin{array}{l}\text { Processo Hie- } \\
\text { rarquico de } \\
\text { análise (HPA) }\end{array}$ & $\begin{array}{l}\text { Estudos que realizaram do método } \\
\text { de HPA para a análise de seus resul- } \\
\text { tados. }\end{array}$ & 4 & 0,1290 \\
\hline Lógica difusa & $\begin{array}{l}\text { Estudos que investigam o uso dos } \\
\text { recursos hospitalares. }\end{array}$ & 4 & 0,1290 \\
\hline $\begin{array}{l}\text { Balance Score- } \\
\text { card (BSC) }\end{array}$ & $\begin{array}{l}\text { Estudos que se utilizam do BSC para } \\
\text { chegar em determinados resultados. }\end{array}$ & 4 & 0,1290 \\
\hline Frameworks & $\begin{array}{l}\text { Estudos que utilizam da elaboração } \\
\text { de frameworks para compor suas } \\
\text { análises. }\end{array}$ & 3 & 0,0967 \\
\hline
\end{tabular}

Fonte: Dados da pesquisa. 
É possível observar de acordo com a tabela 12, que as categorias relacionadas ao método que apresentam maior frequência são questionário estruturado, análise envoltória de dados e processos hierárquicos de análise, isso demonstra uma tendência ucaráter qm quantitativo nos estudos presentes no portifólio.

\section{CONSIDERAÇõeS FINAIS}

Este estudo teve como objetivo a caracterização da produção científica sobre indicadores de desempenho para gestão hospitalar através de análises de artigos publicados entre os anos de 2007 e 2017. Para realizá-lo adotou-se a técnica da análise bibliométrica para o estudo de produção, autoria e publicação, análise das citações, análise das referências e análise de conteúdo, em um portifólio de artigos conforme os métodos utilizados por Silva e Miranda (2018), Pereira Jr., D’avila e Pereira (2018) e Longaray et al. (2018).

Esta pesquisa proporcionou a demonstração de diversos achados que contribuem para a literatura acerca de indicadores de desempenho em organizações hospitalares. Pode-se dizer que o uso de indicadores de desempenho na gestão hospitalar apresenta-se com um tema a ser explorado devido o fato de nenhum dos artigos presentes discorrer com especificidade acerca do tema, de modo que o termo "indicadores de desempenho" sequer aparece em algum dos títulos, neste sentido, indicadores de desempenho são abordados como ferramenta gerencial na avaliação de desempenho hospitalar e não como tema de pesquisa.

Destaca-se que ao identificar os periódicos, os temas e as metodologias mais frequentes, através deste trabalho, apresenta-se um caminho para os pesquisadores que decidem embarcar em pesquisas na mesma temática. Outro ponto de destaque é a caracterização do comportamento dos pesquisadores da área no que diz respeito a fundamentação teórica dos artigos, através da análise das 3.165 citações nas referências bibliográficas presentes nos 31 artigos, é possível verificar neste trabalho quais são os autores mais procurados pelos pesquisadores na hora de fundamentar os trabalhos na temática da pesquisa, assim como os tipos de obras utilizadas e o período de tempo em que as mesmas se encontram. 
Dentre os achados desta pesquisa cabe ressaltar que o tema de maior recorrência nos artigos foi a avaliação do desempenho na área da saúde e que a maioria dos artigos foi escrito por um conjunto de 3 autores espalhados por 5 continentes diferentes sendo a Europa o continente com maior número de autores. Por fim, ressalta-se os achados do estudo da analise das referências bibliográficas, que demonstraram que Norton, D. P. e Kaplan, R. S são os autores mais utilizados no momento em que os pesquisadores vão fundamentar teoricamente seus trabalhos e que o uso de artigos publicados em periódicos supera o uso de outros tipos de obras nesta mesma situação.

Cabe ressaltar que este trabalho refere-se apenas as áreas do conhecimento que foram levadas em consideração na hora das buscas dos artigos, o que é classificado como limitação pois há, em outras áreas do conhecimento, trabalhos com relação á gestão hospitalar, assim como, em revistas internacionais que não possuem classificação de estrato Qualis Capes que poderiam ser levadas em consideração. Outras limitações desta pesquisa dizem respeito ao período de tempo específico para análise e a busca em apenas duas bases de dados. Como sugestão para pesquisas futuras, podem ser desenvolvidas pesquisas na mesma temática, envolvendo maior número de bases de dados, assim como a busca em campo dos indicadores utilizados por gestores.

\section{REFERÊNCIAS}

AKSEZER, C. S. Reliability evaluation of healthcare services by assessing the technical efficiency. Service Industries Journal. v. 31, n. 14, p. 2309-2319, 2011.

ALOLAYYAN, M. N.; ALI, K. A. M.; IDRIS, F. The influence of operational flexibility on hospital performance results: a structural equation modelling (SEM) approaches. International Jornal of Services and Operations Management, v. 13, n. 4, p. 478-497, 2012.

ARAÚJO, R. F.; ALVARENGA, L. A bibliometria na pesquisa científica da pós-graduação brasileira de 1987 a 2007. Encontros Bibli: revista eletrônica de biblioteconomia e ciência da informação. v. 16, n. 31, p. 51-70, 2011.

BARDIN, L.; Análise de conteúdo. São Paulo: Edições 70, 2011.

BRASIL. Constituição (1988). Constituição da República Federativa do Brasil. Brasília, DF: Senado Federal: Centro Gráfico, 1988, 292 p.

BRIGNALL, S.; BALLANTINE, J. Performance measurement in service businesses revisited. International Journal of Service Industry Management, v. 7, n. 1, p. 6-31, 1996. 
CALDAS, M. P.; TINOCO, T. Pesquisa em Gestão de Recursos Humanos nos anos 1990: um Estudo Bibliométrico. Revista de Administração de Empresas - RAE, v. 44, n. 3, p. 100-114, 2004.

DUSSAULT, G. A gestão dos serviços públicos de saúde: características e exigências. Revista de Administração Pública, v. 26, n. 2, p. 8-19, 1989.

GIL, A. C. Como elaborar projetos de pesquisa. São Paulo: Atlas, 2010.

KAPLAN, S R.; NORTON P. D. A estratégia em ação: balanced scorecard. (4⿳亠丷a edição). Rio de Janeiro: Campus, 1997.

LONGARAY, A. A. et al. Propostas de redesenho de processos e o papel das equipes organizacionais: uma análise da produção científica à luz da bibliometria, Sistemas \& Gestão, v. 13, n. 2, p. 246-254, 2018.

LOVAGLIO, P. G.; VITTADINI, G. The balanced scorecard in health care: a multilevel latent variable approach. Journal of Modelling in Management. v. 7, n. 1, p. 38-58, 2012.

MINTZBERG, H. Criando Organizações eficazes: Estrutura em cinco configurações (2aํ edição). São Paulo: Atlas, 2003.

ORGANIZAÇÃO MUNDIAL DA SAÚDE (OMS), 2019. Disponível em https://www.who. int/ Acesso em 24/01/2019.

PEREIRA JR., E. F. Z.; D’AVILA, L. C. \& PEREIRA, F. S. Indicadores de desempenho em serviços e produção: Proposta de mapeamento da produção científica à luz da bibliometria. Sinergia, v. 22, n. 2, p. 61-73, 2018.

PETRI, S. M. Modelo para apoiar a avaliação das abordagens de gestão do desempenho e sugerir aperfeiçoamentos: sob a ótica construtivista. Tese (Doutorado em Engenharia de produção) Universidade Federal de Santa Catarina, Florianópolis, SC, Brasil, 2005.

RAMOS, M. C. A et al. Performance evaluation of hospitals that provide care in the public health system, Brazil. Revista de Saúde Pública, v. 49, n. 43, 2015.

RICHARD, P. J. et al. Measuring Organizational Performance: Towards Methodological Best Practice. Journal of Management, v. 35, n. 3, p. 718-804, 2009.

SANTOS, N. A; LIMA, S. C.; MARTINS, G. A. Análise do Referencial Bibliográfico de Dissertações do Programa Multiinstitucional de Pós-Graduação em Ciências Contábeis (UFPB, UFPE, UFRN E UNB). Anais do XXXIII Encontro da ANPAD (EnANPAD), São Paulo, SP, Brasil, 16, 2009.

SANTOS, M. M. O.; QUEL, L. F.; VIEIRA, A. M.; ROSINI, A. M. Indicadores de Desempenho e Engajamento Profissional em Organizações Inovadoras. Revista de Gestão e Secretariado, v. 10, n. 1, p. 192-212, 2019.

SCHOUT, D.; NOVAES, H. M. D. Do registro ao indicador: gestão da produção da informação assistencial nos hospitais. Ciência \& Saúde Coletiva, v. 12, n. 4, p. 935-944, 2007.

SILVA, A. N.; MIRANDA, A. D. Gestão do conhecimento no setor público: um estudo sobre os artigos publicados em periódicos nacionais no período 2005-2015. Encontros Bibli: revista eletrônica de biblioteconomia e ciência da informação, v. 23, n. 52, p. 73-83, 2018. 
SILVA, M. Z.; BORGET, A.; SCHULTZ, C. A. (2009) Sistematização de um método de custeio híbrido para o custeamento de procedimentos médicos: uma aplicação conjunta das metodologias ABC e UEP. Revista de Ciências da Administração, v. 11, n. 23, p. 217-244, 2009.

SOUZA A. A et al. Indicadores de Desempenho para Hospitais: Análise a partir dos dados divulgados para o público em geral. Anais do $10^{\circ}$ Congresso USP de Controladoria e Contabilidade, São Paulo, SP, Brasil, 15, 2010.

WORMELL, I. Informetria: explorando bases de dados como instrumentos de análise. Ciência da Informação, v.27, n. 2, p. 210-216, 1998.

VANTI, N. Da bibliometria à webometria: uma exploração conceitual dos mecanismos utilizados para medir o registro da Informação e a difusão do conhecimento. Ciência da Informação, v. 31, n. 2, p. 152-162, 2002.

VIEIRA, A. M. Cultura organizacional em instituições de ensino: mapeamento e análise descritivo-interpretativa da produção acadêmica (1990-2005). 2007. 237f. Tese (Doutorado em Educação) - Universidade Estadual Paulista (UNESP), Marília, 2007.

\section{Alisson de Moraes Bastos}

Universidade Federal do Rio Grande

Graduando em Administração - FURG

alissonbastos@furg.br

\section{Samuel Vinícius Bonato}

Universidade Federal do Rio Grande

Doutor em Engenharia de Produção - UFRGS

svbonato@furg.br

\section{Débora Gomes de Gomes}

Universidade Federal do Rio Grande

debora_furg@yahoo.com.br

Recebido em: 15-2-2019

Aprovado em: 16-12-2019

Avaliado pelo sistema double blind review.

Editor: Coordenação do PPGA/UMESP

Disponível em http://mjs.metodista.br/index.php/roc 